\title{
A Review of Men's Underwear Styles and Its Various Fabrics
}

\author{
Dibyendu Bikash Datta* \\ Department of Fashion Management Studies, National Institute of Fashion Technology, Kolkata, India
}

Received: 畫 June 07, 2018; Published: 眥June 13, 2018

*Corresponding author: Dibyendu Bikash Datta, Department of Fashion Management Studies, National Institute of Fashion Technology, Kolkata, India, Email: dbdatta@yahoo.com

\begin{abstract}
Underwear is an innerwear, normally worn next to the skin or under other clothing. The innerwear is also called second skin because it is in direct contact with the skin. Owing to the importance of this aspect, the fabric of the innerwear is the next important criteria that are considered while buying innerwear. Innovations and technology are constantly evolving for men's underwear industry. Underwear is essential for hygiene and some styles provide support in regulating the body's shape. Social situations, weather conditions, and the apparel can affect the style of undergarment men wear. Different types of men's underwear and the variations on each of the styles are widely available. The availability of products related to intimate apparels for men available online is expected to drive the growth of online shopping for underwear worldwide. This paper describes the various styles of underwear garments and the fabric they are made from.
\end{abstract}

Keywords: Underwear; Innerwear; Comfort; Intimate; Briefs

\section{Introduction}

Men are no longer living in an age where their un dies have to be ugly and practical. It has become a billion dollar industry which has added focus to fashion, comfort, and gender appeal. Even though the underwear is hidden most of the time that does not negate the role of an undergarment [1]. Men, in general, avoid exploring the possibilities and are in a habit of sticking to the usual style of underneath article. However, over the past few years, the intimate apparel industry has definitely seen a lot of progress. The classic debate over 'boxer versus brief' has now shifted to the usability of the erotically attractive styles of men's underwear. These days the customers are open to discussing the skivvies like lace underwear and pouch underwear for men which were earlier considered a taboo and stigma. The underwear industry for men is now flooded with choices that range from the classic styles of boxer briefs and boxer shorts to the radically aesthetic men's thongs, G-strings, and even C-string. It won't be wrong to state that this is one category of men's apparel, which initially held the reputation of clothing that just covers the genital, has come a long way to being an essential staple for defining the overall style quotient [2].

Comfort, style, and color are the three attributes which need to be considered for men's underwear. Comfort is a fundamental necessity. The comfort of the underwear is related to three things; the size, climate, and fabric used. Underwear that adapts to body's temperature whatever the climate provides a better comfort by keeping warm in winter, cool and dry in summer [1-3]. Generally, underwear is measured with designations of S, M, L, and XL (Table 1) below shows the general waistline measurements in inches according to the size.

Table 1: Standard undergarment sizes for men.

\begin{tabular}{|c|c|c|c|c|c|c|c|c|}
\hline Size & S & M & L & XL & XXL & 3XL & 4XL & 5XL \\
\hline Waist (inches) & $28-30$ & $32-34$ & $36-38$ & $40-42$ & $44-46$ & $48-50$ & $52-54$ & $56-68$ \\
\hline
\end{tabular}

Small (S); Medium (M); Large (L); Extra Large (XL). 


\section{History of Men's Underwear}

According to the historians, the oldest example of men's underwear, the leather loincloth, dates back to the cave man in early 3000 BC [4]. The loincloth is the simplest form of underwear that is draped around the hips and groin. This was the first time when men were observed wearing anything other than a fig leave to cover up their privates. This remained in the trend for several years. The only change was in terms of fabric used in making of this apparel. The variation on the loincloth seems to have persisted when loosefitting trousers called the braises came into fashion. The trousers tied at the waist and thighs were a vogue into the middle ages [5]. These were later upgraded into codpieces in the 16th century. These underneath apparel were a fashion statement and usually came in bright colours and with padded contraptions [6].

The fitted knee-length apparel that men wore beneath their pants was not very comfy. This led to the invention of the elastic waistband in the year 1925 and this led to the development of boxer shorts. Thus, the underwear for men become much more resilient and supportive [7]. 'Look good... Feel good... Be twice the man in Y-front', was the quote that described the very first men's brief (Figure 1). This came in as a pleasant surprise for the male population who needed something lot more supportive down there [8]. However, it is lesser known fact that boxer shorts were not the very first invention in men's underwear industry. Jockstraps were invented way before in the late 1800s. This skivvy style was specifically invented to provide comfort and support to the cyclists [9]. Pioneered by the designer, John Varvatos, boxer briefs were introduced in the year 1990. Combining the length of the boxers and the snug fit of brief, this underneath article took the entire industry by storm. The aesthetically revolutionary underneath article is till date the most used style [10].

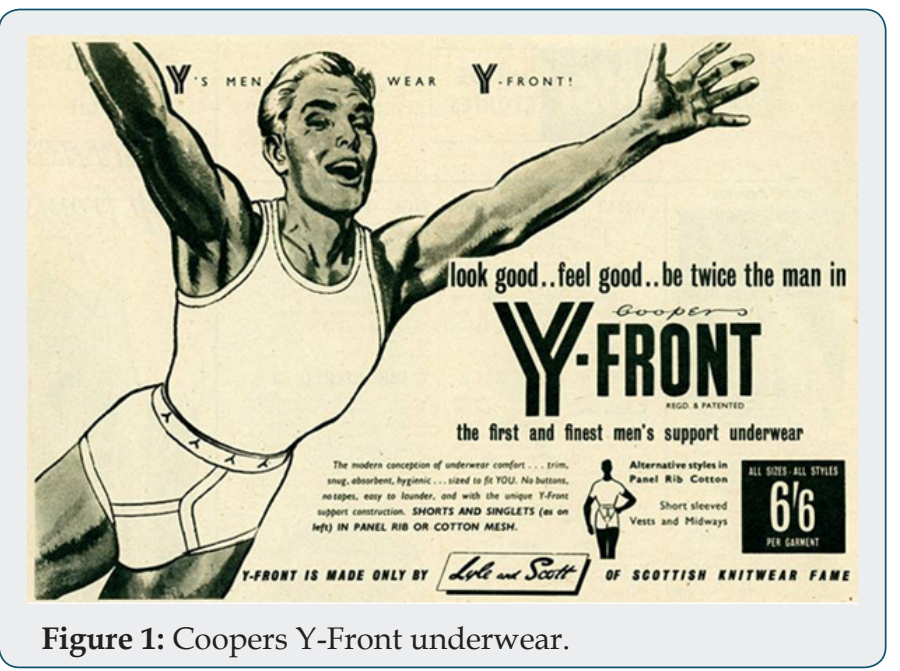

\section{Men's Underwear Styles}

Underwear is available in different styles and the majority of men generally prefer wearing a particular style no matter the activity. Long gone are the days when the choice of men's underwear was simple and limited. Despite the fact, the underwear is a lesser talked about subject in menswear; the options available in this category these days are ample. It makes more sense to wear a different style when travelling, working out at the gym or when wearing trim denim or trousers instead of wearing the same style for all day every day. The different aspects of the various styles of men's underwear are given in (Figure 2).

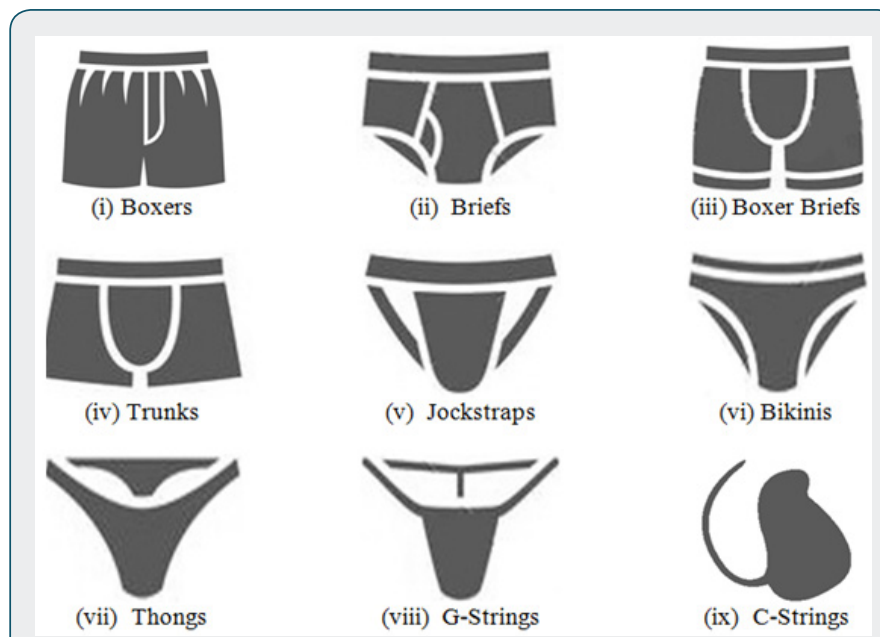

Figure 2: Men's underwear styles.

\section{Men's Boxers}

The boxer shorts were initially introduced in 1925 and were known as 'shorts for boxers' [4]. This was the first time when elastic waistbands were used in the undies. Even though it took some time to gain popularity, the underwear style is true to its namesake. Inspired by the actual boxers, the skivvy extends a few inches above the knees covering almost one-third of the thighs and has a contoured pouch for comfort. However, they lacked the support that the union suits offered. The problem of bunching and riding up highlighted the need of undergarment that fitted the body snugly. However, the baggy style kept everything airy and comfy. Despite the change in the need and preference of men as well as the presence of a number of underwear styles, boxers are of the most popular underneath article till date. However, the modern boxers are mostly designed in form-fitting styles that hug the wearer snugly. Mostly boxer shorts used to have a fly opening in front. Some of the undies had metal snaps while others had buttons. However, the trends have changed and the modern designs do not have fastening mechanism to close up the fly. Above all, a comfy style is a perfect option for the laid-back evenings and weekend plans.

\section{Types}

Lace Boxer: The masculine style combined with the tender touch of lace is for all those who like to push the boundary of their style. Keeping the wearer bang on trend, the flimsy fabric keeps the crotch airy and dry all day long.

Sheer Boxer: Taking the conventional underneath article to the new height of sensuality, the sheer boxers for men allows flaunting 
a lot of skin. With the same support, these boxers add a voluptuous edge to sex appeal.

Pouch enhancing boxers: The un dies provide enhancement contraption to the wearer. The pouch lifts the manhood and enhances the visibility of the manhood. Unlike the padded underwear, these skivvies provide natural looking bulge.

Benefits: The first and the foremost advantage of the baggy boxers is the fact that they are extremely comfortable. The loose fit allows airflow. With ample ventilation, the underwear is a perfect option for everyday use. The traditional un dies with a touch modern pouch enhancing technology elevate the position of the entire package. The abrasion-free pouch reduces chafing and sweat secretion. As the sweat retention reduces, the skin problems such as rashes, itchiness, and irritation reduce gradually. Along with comfort, the contemporary styles boxer shorts even offer best ever support to the manhood.

\section{Men's Briefs}

Inspired by bathing suits, the Y-shaped snug-fit undies were introduced in the year 1930s [4]. The practical approach and the form-fitting style took over the market in no time. Unlike the boxer shorts, the underneath article held the genitals in a relatively fixed position. The increased support and alluring appeal have occupied a section of the top drawer of every man since then. In the 1980s, the conventional un dies were upgraded to fashion briefs. This was marked by the presence of bright colors and flashy prints. Tight briefs have always been blamed for infertility in men. However, it is not the underwear cut that leads to the medical issue. Choosing the wrong size and incorrect fit of undies lead to squashing which ultimately causes low sperm count. The subtly sexy apparel covers the entire rear and package but leaves the upper thighs and the side of the leg for the show. Thus, the undies provide the muchneeded sex appeal, but without going over-the-top. Men's briefs are available in low, mid and high rise cut. Thus, one can make their choice according to the style of their bottoms. Despite the fact that the comparison between boxers and briefs seem to be neverending, the experts sum up by saying that the low sperm count is an after effect of overheating of testicles. The underneath articles that bundle the package too close to the body of the wearer raises the temperature of the same. Thus, both the styles are equally good, if the right size is chosen. It is a versatile underwear style perfect for all occasion, event and season and preferred undies for the workplace. The high cut will keep it breezy while the contouring pouch keeps the junk together. The supportive styles of the appealing briefs are perfect for getaways and adventures trips. Even though the boxer briefs are preferred options for vigorous physical activities, the formal one completes the ensemble.

\section{Types}

High-rise brief: This is the traditional style of the brief that sits right below the belly button and above the natural waistline. Worn with high rise pants and jeans, it hugs the package and pulls it up.

Mid-rise brief: As the name suggests, the mid-rise briefs for men sits in between the low-high and high-rise briefs. With the same support and comfort, it is a much versatile option for those who don't like their waistband popping out of the trouser.

Low-rise brief: This style was introduced in the year 1990s when low-rise jeans and trousers came in fashion. Low-rise briefs sit below the natural waistline on the hips, the underwear prevents sagging.

Lace brief: With the aim of catering to the wildest fantasies of men, fashion connoisseur has stylized the traditional undies with a contemporary fabric. Lace briefs for men are one such option that can help to flaunt the wild side without negotiating comfort.

Sheer brief: The see-through styles of the briefs provide a teasing glimpse of one's skin.

Benefits: The snug-fit style and the high cut of men's brief provide easy leg movement. The undies are a practical option for long working hours as they maintain air circulation in the crotch area. The briefs, these days are designed with modern technology. It keeps the package together and away from the body. This prevents from clashing and reduces the possibility of chafing and other related problems.

\section{Men's Boxer Briefs}

Boxer briefs came into existence in the year 1990 [11]. The boxers were comfy but lagged the much-needed support. Moreover, the loose fit led to bunching up of fabric. On the other hand, with the snug-fit of traditional briefs, comfort suffered to some extent. This led to the evolution of something that had the positives of both the best-selling men's underwear styles. The underwear style, pioneered by John Varvatos, is considered as one of the 'greatest apparel revolutions of the century' providing support of a brief and coverage of a boxer. Boxer briefs are a great choice as an alternative that provides both styles as well as comfort. The, also known as, 'manly' pair of underwear hugs the body of the wearer snugly. The anatomical pouch offers a great deal of support and makes groin look larger. The functional underneath apparel provides a broader space for the male anatomy to breathe freely. The pouch of traditional undies kept the genital somewhere in between the thigh. This led to squashing, chafing and other such issues. The anatomically correct design of boxer brief is till date the most recommended option for those who seek comfort, support as well as freedom down there. The form-fitting style of the undies covers the one-third portion of the thighs without bunching or riding up. The functional underwear style definitely beats the other underwear styles. This underwear style is a staple for trekking or any other adventurous trip. Boxer briefs are an alternative for the sporty jockstrap. If the revealing rear of the jockstraps embarrasses at the locker room, then, these supportive undies are a perfect option. 


\section{Types}

Low-rise boxer brief: This underwear style sits below the natural waistline of the wearer. Thus, it can be donned with low rise jeans.

Cheeky Boxer: This is the cheekiest style of a boxer with the traditional support and conventional coverage in the front, the underwear provides a teasing glimpse of buttocks.

Benefits: As mentioned above, boxer briefs were invented to provide the positives of two of the most used underwear styles, that is, briefs and boxers. The skivvy style provides both body-hugging fit and ultimate support. The traditional boxers with loose fit would ride up the pants of the wearer. The snug fit boxer briefs reduce this problem. The possibility of twisting and bunching reduced making it an apt option to be paired with even the skinniest jeans as well as trousers. Along with the much-needed comfort and support, the versatile boxer briefs work under all types of clothes and can be worn on any occasion. This athletic style is particularly a functional option for workouts and other athletic activities. The boxer briefs are considered to be one of the best alternates for the traditional jockstraps.

\section{Men's Trunks}

The trunk has been used as a swimwear for men since the 1940s [12]. This underneath article replaced the bulkier swimsuits that covered the torso. This apparel was heavy as well as hot and hence the stylish cut of trunk came in as a pleasant surprise for the style-conscious male population. The trunk features loose fit undies mostly worn for sports purpose by boxers and swimmers. However, over the past few decades, this style has been accepted as one of the most functional, yet stylish men's underwear. The trunk underwear is now designed to fit the wearer snugly and has conventional all round coverage and anatomically correct pouch. Despite being in the underneath apparel for a very long time, the trunks celebrated their heyday in the 1990s. After tasting success in the swimwear category, it later turned out to be a staple in the underwear drawer of every man who has higher regards for comfort and support along with style. The modern trunks offer a bit more coverage than the boxer briefs. However, the support and comfort are as good as any other underwear style. The pouch of the underwear style is designed to keep the package away from the body of the wearer. In many countries, the trunk is used to describe swim briefs. This underneath apparel is best for sports and water adventures. The form-fitting styles of trunk underwear can be used as an everyday wear as well since it keeps cozy at the workplace.

\section{Types}

Sports Trunk: Other than the swim trunk, there is a range of sports trunk that offers the support and lifts to the manhood while undergoing any sort of physical activity. Most of these undies are made up of mesh cotton fabric that keeps it airy. So, the irritation of sweaty crotch is not a problem anymore.
Shaping Trunk: Some of the pieces in this category are perfect shape wear apparel that can help stay in shape. The compression shorts are made of airy fabric that keeps airy while redefining one's personality.

Benefits: The versatile piece can be worn as swimwear as well as underwear. The supportive and comfy underneath apparel is perfect for every occasion and purpose. The shaping trunks are a cozy way of staying in shape. The sleek style can be worn with formal or casual wear. It slips in with any of the shirt or t-shirts without the visible linings.

\section{Men's Jockstraps}

The jockstrap underwear has been in use since 1888 and for good reason. The invention of bicycles highlighted the need of underwear that would prevent the cyclist from the saddle sore. Jockstraps offered less restriction and offered more support. Not only this, the pouch of the underwear featured protective cups. Initially, these cups were made of hard plastic or steel, perforated for ventilation. The heavy material was protective, but inconvenient and led to overheating of testicles. Later the protective shield was made of soft and flexible material [13]. In recent past, this style has shifted gears and has become an erotic piece for the pleasure purpose. The fashion jockstraps are designed to bring the male genital forward. The cheeky style of men's jockstrap covers and supports the front, but leaves nothing to the imagination at the back. This keeps breezy down there while working out or undergoing any sort of strenuous activity. The revealing back gives this style an edge over all the other undies. However, the ample support on the front makes it one of the most prominent options for the rigorous physical activity.

Some jockstraps are athletic and fashionable. The racy style, sensual details and body-defining fabric of the fashion jockstrap can spice up intimate affairs. The variant handsomely highlights the masculinity and gracefully pleases the sense. The sports jock accompany from early morning jogs to the workout sessions. It is specifically designed for the athletic personalities. Whether on the soccer field or at the gym, these undies can protect and pamper the manhood. The erotic barely-there apparel can even accompany for the date night. The supportive, yet outrageous undergarment counts for the fashion-forward profile on the special occasions. The fast-drying fabric and the package boosting pouch will provide much-desired lift as well as comfort while hiking or trekking. Boxer briefs and jockstraps are two of the best options for such adventurous outings.

\section{Types}

Athletic Jockstrap: The jerk-resistant cup of the underwear finds the support from the built-to-last waistband and straps. Made of lightweight and high-end fabric, these undies serve the practical purpose of keeping crotch dry.

Fashion jockstrap: The revealing cuts coupled with zestful 
patterns, the underneath articles can give a steaming hot look. The fashion jocks are a modish alternative to conventional style for the special occasions. This collection of these undies ranges from feminine lace jockstraps for men to the see-through sheer and mesh jockstrap.

Benefits: Jockstraps, initially, offered cups that acted like a protective shield for manhood. However, the modern jocks provide natural contraptions. The pouch elevates the manhood and keeps it in an elevated position. Thus, the well-constructed undies protect the privates without the inconvenience of pads. The anatomical pouch enhances the visibility of the front profile. The straps at the back add to the support and keep everything in the right place. The rear aids ventilation and keeps it breezy by allowing free air flow. Thus, the undergarment makes sure that the scrotum is dry.

\section{Men's Bikinis}

In the era of boxer briefs and men's brief, bikinis for men was like a rare bird. The underwear style was introduced in the year 1942. After gaining a lot of popularity among women in the 20th century, the erotic style of underneath article was usually used in the swimwear category of men. However, with time it has become popular as men's underwear [14]. Bikinis for men are considered a sensual version of men's brief. This underwear style offers the same fitted look and support as that of the traditional briefs. However, it was skimpier than the former. This style of underneath article was initially used as swimwear. Later it became one of the best sellers in men's underwear category. This is one of the best options for men who are looking sex appeal with going over-the-top. This is one of the most versatile underwear styles that can sport at work as well as for casual spree. The conventional bikinis are an apt option for the long working hours. With the top-notch support and skimpy cut, the underwear holds the package and keeps it breezy. For parties and other casual occasions, the sexy, yet functional styles of pouch bikini underwear are ideal. The pouch elevates the position of genital and accentuates the visibility of the front profile. Thus, one can bring the entire attention in the right place.

\section{Types}

String Bikini: This is the skimpiest bikini available in the intimate apparel category. The skivvy style features a tiny front pouch to hold the entire package together. While the string waistband and revealing rear allows the wearer to flaunt a lot of skin.

Brazilian Bikini: The Brazilian cut was initially popular in the category of women panties. The Brazilian bikinis for men rear cut while the front may feature the usual style. The fabric at the back of this underwear cut stretches a bit to provide more coverage than thongs. The amount of skin that this underneath article style ranges somewhere between a thong and a bikini.

Bikini Brief: The bikini briefs are skimpier than the conventional briefs. The waistband lies below the natural waistline of the wearer and allows a lot of skin show. Keeping the coverage minimal, the undies keep the sex appeal to the next level. However, the underwear offers the support that matches the traditional undies.

Micro Bikini: This is one of the sexiest options for all those who like to keep it sleek down there. The skimpy cut and revealing patterns of the undies can tantalize the wild side of personality. The waistband of micro bikinis rests at or below the natural waistline of the wearer. This can get rid of the waistband popping out of the trouser. It won't be wrong to state that micro bikinis for men are an ideal option when looking for style without revealing the assets.

Benefits: The versatile style of underwear offers many benefits. Men's bikini underwear is perfect for work and pleasure. This is one contemporary style that can be used for the special occasions as well as a daily wear. While most men still hesitate in trying the skimpy cuts like men's thong and G-strings, bikinis are a safe option for them. It can give the band on-trend look without revealing much skin down there. Along with the much-needed sex appeal, these underneath apparel offers comfort and support at par?

\section{Men's Thongs}

The early 1980s saw a radical change in underneath article industry for men. This was the time when one of the sexiest underneath apparel, that is, men's thong underwear was invented [15]. Despite the fact, the thongs are considered as a predecessor of traditional loincloth, the underwear style took some time to enter the wardrobe of the male population. Having gained popularity as women apparel, thongs for men was earlier a taboo. Considered as a feminine style, the underneath apparel has become a staple for all the fashion-conscious men. With ample coverage in the front, the rear features nothing more than a tiny fabric that just covers the butt crack. The sexy apparel feature not fabric at the back, thus, it has no underwear line. The contemporary undies feature special abrasion-free pouch that elevates the position of the manhood. This enhances the visibility of the front profile and provides a natural accentuation. The ultra sexy men's thong is perfect apparel for pleasure purpose. The sports thong can accompany to the gym. The pouch of these skivvies keeps the package together, but away from the proximity of the body. This reduces chafing, sticking and squashing.

\section{Types}

Lace thong: The combination of a thong with see-through lace is a deadly combination. The revealing pattern of a thong with the fragile touch of lace fabric can effortlessly boost a lot of sex appeal to one's personality.

Sports thong: A range of thong that features flair of an athletic look. The pouch of these undies offer extra support to the junk and allow triggering wild side without compromising the sporty personality. 
Brazilian thong: The Brazilian cut at the back of the underwear allows a bit more than expected. The barely-their underwear is for the male population who just settle with anything ordinary.

Sheer thong: Men's thong crafted in the transparent sheer fabric leaves little to nothing to the imagination. The extremely revealing undergarment is for all those who dare to go bold.

Benefits: The incredibly sexy apparel let flaunt the derriere. With minimal coverage, the undies will shoot the sex appeal through the roof. Thongs are perfect for men who seek minimal coverage and ample support. Other than the much-needed support, the skimpy underwear style is a practical option for keeping manhood dry and comfy even during the hottest day. At the same time, it can even effortlessly raise the temperature of the date night. No fabric at the back leads to any nasty underwear line. It allows putting on skinniest jeans without worrying about the visible line. Moreover, the undies feature a thin and sleek waistband that spares the embarrassment of popping waistband. It is a better option for men as it makes laundry a cinch for the lazy fellows. Not only this, the thongs are even a better alternative for going commandos.

\section{Men's G-Strings}

G-strings are considered as the skimpier version of thong underwear. These underwear styles were incorporated into the vocabulary of women's underwear the 1980s. However, with time men got body and style-conscious. With the change in the taste and preference, it gained popularity in the early 2000s [16]. Just like thongs, G-strings for men covers absolutely nothing at the back. However, the front offers ample support to the boys of the wearer. There are a lot of saying regarding the name of the underwear. Some say that it is named after the G-string of a guitar. While according to Wikipedia, in the 19th century, the word G-string was used for a loincloth. In the opinion of linguist Robert Hendrickson, the letter ' $G$ ' stands for the groin. The feminine style of underneath article with a touch of machismo can give a contrasting combination to the masculinity of the wearer. This underneath apparel was specifically used for the pleasure purpose. It still continues to be a staple for all those prefer keeping everything spicy in their relation. The provoking glimpse of the asset can take the sex appeal to the next level. The seamless underwear can be teamed up with a variety of outfit and can be even worn on formal occasion. Paring them with office attire or even tuxedo will get rid of the prominent problem of visible underwear line.

\section{Types}

See-through G-strings: The men's G-string underwear crafted in a see-through sheer fabric is not for the faint-hearts. Covering nothing at all down there, the underneath article can effortlessly spice up intimacy.

Lace G-strings: The soft fabric and breezy cut of the sensual underwear put at ease. The men's lingerie gives a good confidence boost by taking sex appeal to the next level.
Pouch enhancing G-strings: The non-traditional underwear is designed with modern technology. Unlike the padded and push-up underwear, these undies provide natural enhancement contraption. The anatomical pouch provides wearer-specific isolation; the underwear enhances the visibility of the front profile.

Benefits: The sleek style is not just a sensual experience, but is a eye-candy for the partner and puts up a great show on a special night and keep everything excited in the intimate affairs.

Just like any other skimpy style, the barely-there undies provide air circulation and keep the crotch breezy and dry. Thus, it serves a practical purpose for every man who suffers from sweaty manhood.

Along with ample support and comfort, the anatomically correct pouch enhances the visibility and accentuates the profile. This brings the entire attention to the rightful place.

\section{Men's C-strings}

C-string underwear for men is as narrow as G-strings, but it comes without the support of the string waistband. The awe-striking style of the undergarment made people rethink who thought that thongs were unnecessary revealing. Initially, launched for women, the undergarment has caught the attention of fashion-savvy men in the past decade. The underneath article was earlier known as 'Invisible underwear'. The C-shaped undergarment is in the shape of a headband. The entire structure slips right in between the legs of the wearer and appears to be floating in the air. The C-shape of the underwear ensures that it sticks to the body. The men's C-string underwear has a cup-shaped pouch that holds the package together [17]. It finds its support in between the butt-cheek of the wearer. The internal frame holds everything lifted and keeps it in the right position. The entire structure is self-supportive and covers the basic essentials of the wearer. The pouch accommodates the male anatomy without any unwanted strap. C-string is an ideals style of men's underwear for tanning during sunbathing and prevents the tan lines. The invisible underneath article provides the coverage where need it the most, rest everything is left for the show. Pairing with tight trousers or jeans is necessary, so that, the C-string stay in the right place.

Benefits: The erotic undies are tailored for experiencing the sexiness and best ever intimacy. The sexy garment is very comfy, but not in the beginning. However, once used to it, one can wear it at every occasion only if the wire is tight against the body between the cheeks.

\section{Men's Underwear Fabrics}

Earlier, cotton was the only fabricused in making undergarments. However, with changing preference and technological advancements, the underwear industry for men has seen a lot of innovation in terms of fabric. There are a plethora of options in this category. Some of the fabrics mostly used are cotton; nylon, modal, rayon, polyester, and silk. These are blended with spandex in the 
right proportion to ensure quality, comfort, and durability along with ample flexibility. Lycra, elastane are specifically used to add stretchiness to the fabric of the undergarment [4]. A brief discussion about the various fabric options in the underwear category for men is described below.

\section{Cotton Underwear}

Considered as the king of fabrics, the natural fiber is one of the most used fabrics in men's underwear industry. This was, in fact, the only fabric used in making the underneath apparel for a very long time. However, the last couple of decades has seen a plethora of options in this category.

\section{Properties of cotton}

Cotton is very soft and lightweight. Thus, the underneath clothing made up of this fiber is comfortable. The fabric has tiny pores that allow breathing well. Keeping the ventilation and air circulation to the next level, cotton underwear provides a comfy feel down there. Cotton has high absorbency and is moisture resistant. Thus, it can keep dry even during the hottest day of summer. The fabric is stronger when wet than dry. Moreover, it can withstand heat, detergent and even bleach. This ensures the durability of the underneath article. However, it is more prone to shrinkage if not treated properly [18].

\section{Best wearing condition}

The undies crafted in high-quality cotton is perfect for all occasion, event and season. However, the fiber is slow drying, so, washing it is sometimes inconvenient during winter. Fabrics like silk and wool is a preferred option for this season. Use of cotton underwear should be avoided while exercising as they may lead to abrasion. Nylon is a better option on such occasion.

\section{Nylon underwear}

Nylon is the best alternative for cotton. It is a generic designation of the family of synthetic polymers. Derived from diamine and dicarboxylic acid, the polyamide fiber is the second most used fabric in underwear industry for men.

\section{Properties}

The strength of the nylon makes the undergarments made of this fabric durable. The tenacity of nylon is not gone even with age. The color retention property of nylon provides it an edge over cotton. The fabric retains colors of the clothing even after multiple washes. The smooth texture of the fabric has excellent abrasion resistant. This is the reason why it is used in swimwear and swim costumes. The heat conductivity of the fiber depends on the constructions. However, it still disallows the heat to pass through it, thus, keeps the wearer warm during the cold season. Nylon has low absorbency and hence dries quickly. This makes the nylon underwear perfect for those who suffer from the sweaty crotch [19].

\section{Best wearing condition}

Nylon underwear is best for hitting the gym or for other rigorous exercises. These undies dry easily and reduce the possibility of crotch rot due to moisture retention. This is even a preferred option for the water adventures and beach outing. The swimsuit crafted in nylon keeps manhood cozy and abrasion-free when taking a plunge into the water.

\section{Modal underwear}

There is no fabric softer than modal in the undergarment category for men. In fact, this material is around fifty percent more water absorbent than cotton. Modal is a type of rayon and the second generation regenerated cellulosic fiber.

\section{Properties}

Modal is the softest fabric in the world. This pretty much explains its significance in the men's underwear industry. The undies made of this fiber can keep cozy all day long. Despite the soft and silky feel, the material wears resistant and strong. This is one fabric that has very high wet strength, thus, it can even withstand machine wash and can be tumble dried. Even after multiple washes, the material stays absorbent, soft and supple. Modal is often blended with cotton and other synthetic fibers. It even allows easy dyeing [20].

\section{Best wearing condition}

This is a perfect option for summer wear. The lightweight fabric can keep dry and cozy during the hot and humid weather. It can be used for everyday use. However, avoid wearing them during workouts and other athletic activities. Even though the wet strength is an alluring property of the fabric, this is not an apt option for the bikini season. Nylon and polyester are some of the preferred options for the fun-filled season.

\section{Rayon underwear}

Rayon has many properties similar to cotton and other natural fibers. This was a commonly used fabric in summer clothing for both men and women. It is now being used in making underwear for men as well.

\section{Properties}

Just like cotton, rayon is comfortable, airy and moisture absorbent. Thus, the undies made up of this fabric are a perfect choice to deal with excessive sweating and problems related to it. What makes it versatile is the fact that it can be easily dyed in vivid colors. This has made the introduction of some jazzy and lesser used shades possible in the intimate category for men. The fabric has moderate strength but is soft to the skin and abrasion resistant. Thus, it is practical option to reduce sticking and squashing of the manhood during the day. The shrink resistant material makes the underneath article durable, and sustain multiple washes. Rayon 
underwear materials to be washed by hand and should not be clubbed with other clothes in the washing machine [20].

\section{Best wearing condition}

The lightweight fabric is an apt option to beat the heat of summer. The thin material provides top-notch ventilation and keeps everything breezy down there. The material stretches easily, so, it is not a recommended option for all day use. Moreover, it won't provide the much-needed warmth during winter.

\section{Polyester underwear}

Polyester includes naturally occurring chemicals. It is often blended with natural fibers such as cotton that helps bind the fibers together.

\section{Properties}

Polyester provides a smooth and synthetic feel. The skivvies made of this material feel very soft against the skin of the wearer. The fabric is resistant to wrinkle, mildew as well as abrasion. This is why it is used in making men's swimwear. The fabric keeps the package isolated and away from the body even when drenched. Polyester is extremely strong and does not stretch or shrink. Thus, these undergarments last longer than usual undies. Moreover, it can be machine washed and dried [21].

\section{Best wearing condition}

As mentioned above, it is an apt option for the bikini season. The swimsuit crafted in polyester will facilitate all sorts of water adventures. The material has high absorbency; hence, it is not a recommended option for the hot season.

\section{Silk underwear}

The smooth and soft texture of silk provides luxurious feel down there. A filament fiber formed from the protein secreted by silkworms is known for its shiny look, softness as well as insulating power.

\section{Properties}

The silky feels and sleek style of the fabric make it a perfect material for making the novelty underwear. The extremely soft texture keeps the wearer comfortable. Insulation is a unique property of silk. It is very absorbent and not a good conductor of heat. It traps the heat and prevents it from leaving the clothing. Thus, it creates an insulating layer in between the cloth and the body of the wearer. Silk is durable and has the greatest pull strength as compared to all the natural fibers [22].

\section{Best wearing condition}

As silk is a bad conductor of heat, thus, it is the perfect option for winter. The insulating layer will prevent the heat from escaping the body and will keep warm. Silk doesn't provide as much support as cotton and other materials. Hence, it is not a suitable option for all day use and is meant for special occasions.

\section{Elastane}

Elastane fibers are made of polyurethane and commonly known under the trade names Lycra and Spandex. Elastane is often used in applications where a permanent elasticity is necessary as it has the ability to provide the desired elasticity in knitted fabrics with a low percentage blend. High elasticity even in low concentration blends. In sportswear, almost all garments have a blend containing elastane since it enables freedom of body movement by reducing the fabric resistance. The use of elastane blends results in difficulties when recycling garments since it is necessary to separate the different fibers. Elastane is considered to be an environmentally unfriendly fiber because of its production where non-organic and hazardous solvents are used [24].

\section{Conclusion}

Comfort is the most important property for underwear. Men tend to prefer cellulosic-based fabrics in underwear. Synthetic fabrics are often perceived as sportier as and cooler than cellulosic-based fabrics and are highly associated with sporty, good breathability, high quality, and good support, comfortable and stay dry. India is moving from plain cotton underwear to designer vest with advanced fabrics that serve the purpose of active wear and follows some trends and style. The new emerging trend in the innerwear industry is the variety of fabric that is used for the underwear. People prefer these fabrics due to comfort as well as the fit of the same. Stretchable underwear of perfect fit and style are available in the Indian market and manufacturers are planning to expand by looking at the growing segment of the innerwear market. With some of the modern technologies being incorporated in the innerwear segment, the future of this industry looks promising.

\section{References}

1. Hale R, Hodges N (2013) Men's branded underwear: an investigation of factors important to product choice. Qualitative Market Research: An International Journal 16(2): 180-196.

2. Kremer K (2016) US Patent Application 101.

3. Slater K (1977) Comfort properties of textiles. Textile progress 9(4): 1-70.

4. Cole S (2012) The story of men's underwear. Park stone International.

5. Cunnington CW, Cunnington P (1992) The history of underclothes. Courier Corporation.

6. Bowman K (2016) Corsets and Codpieces: A History of Outrageous Fashion, from Roman Times to the Modern Era. Sky horse Publishing Inc pp. 176.

7. Gray ML (1925) US Patent. Patent and Trademark Office. Washington, DC: USA.

8. Jobling P (2003) Underexposed: Spectatorship and Pleasure in Men's Underwear Advertising in the Twentieth Century 26(1-2): 147-162.

9. Stamford B (1996) Sports bras and briefs: choosing good athletic support. The Physician and sports medicine 24(12): 99-100.

10. Klein C, Sport CK (1970) Calvin Klein Info/Shopping, Women's Apparel. Early years 1(1-3): 1980s-1985.

11. Le Ann MT (1990) US Patent. Patent and Trademark Office. Washington, DC: USA. 
12. William H (1940) US Patent. Patent and Trademark Office. Washington, DC: USA.

13. Rust TE (1977) A history of swimwear reflecting some sociological and technological changes (Doctoral dissertation, Virginia Tech). Cox C G-String and Thong.

14. Opiyo V (2017) The G-String as a Space for Sexual and Political Imagination: Rethinking Discourses of Youth, Power and Globalization in Kenya. Research on Gender and Sexualities in Africa 77.

15. Scott L (2010) Lingerie: A Modern Guide. A and C Black.

16. Stanković SB, Popović D, and Poparić GB (2008) Thermal properties of textile fabrics made of natural and regenerated cellulose fibers. Polymer Testing 27(1): 41-48.

17. Hearle JW, Morton WE (2008) Physical properties of textile fibers $4^{\text {th }}$ edn. Elsevier.
18. Gun AD (2011) Dimensional, physical and thermal comfort properties of plain knitted fabrics made from modal viscose yarns having microfibers and conventional fibers. Fibers and Polymers 12(2): 258-267.

19. Yamashita H, Nakano Y (2008) Polyester: properties, preparation and applications. Nova Science Publishers, Incorporated.

20. Corazza M, Borghi A, Minghetti S, Toni G, Virgili A (2015) Effectiveness of silk fabric underwear as an adjuvant tool in the management of vulvar lichen simplex chronicus: results of a double-blind randomized controlled trial. Menopause 22(8): 850-856.

21. Lin JH, Chang CW, Lou CW, Hsing WH (2004) Mechanical properties of highly elastic complex yarns with spandex made by a novel rotor twister. Textile Research Journal 74(6): 480-484.
To Submit Your Article Click Here:

This work is licensed under Creative Commons Attribution 4.0 License

DOI: 10.32474/LTTFD.2018.02.000134

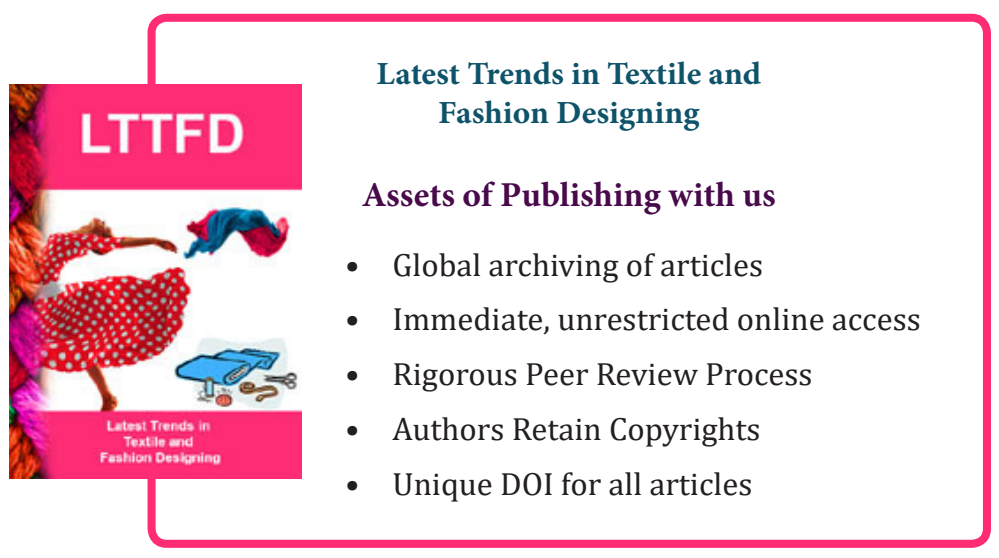

